\title{
Analytical study of heat transfer of a unsteady newtonian nanofluid flow problem
}

\author{
A. EL HARFOUF ${ }^{*}{ }^{1}$, A. WAKIF ${ }^{2}$ AND S. HAYANI MOUNIR ${ }^{\mathbf{1}}$. \\ ${ }^{1}$ Sultan Moulay Slimane University of Beni Mellal, Polydisciplinary Faculty of Khouribga, Research Team \\ of Energy, Materials, Atomics and Information Fusion, BP : 145 Main Khouribga, 25000, MOROCCO. \\ ${ }^{2}$ Hassan II University, Faculty of Sciences Aïn Chock, Laboratory of Mechanics, B.P.5366 Mâarif, \\ Casablanca, MOROCCO.
}

Abstract: Heat transfer behavior of unsteady flow of squeezing nanofluid (Copper+water) between two parallel plates is investigated. By using the appropriate transformation for the velocity and temperature, the basic equations governing the flow and heat transfer were reduced to a set of ordinary differential equations. These equations subjected to the associated boundary conditions were solved analytically using Homotopy Perturbation Method and numerically using Runge-Kutta-Fehlberg method with shooting technique. Effects on the behavior of velocity and temperature for various values of relevant parameters are illustrated graphically. The skin-friction coefficient, heat transfer and Nusselt number rate are also tabulated for various governing parameters. The results indicate that, for nanofluid flow, the rates of heat transfer and velocity had direct relationship with squeeze number and nanoparticle volume fraction they are also a decreasing function of those parameters.

Keywords: Heat and Mass Transfer, Nanofluid, Homotopy perturbation method, Runge-Kutta-Fehlberg, Received: August 14, 2020. Revised: December 4, 2020. Accepted: December 24, 2020. Published: December 31, 2020.

\section{Introduction}

The word nanofluid represents the fluid in which particles of size with order of nanometer (diameter $<100 \mathrm{~nm}$ ) are mixed in the base fluid. The nanoparticles used in nanofluids are generally made of metals $(\mathrm{Al}, \mathrm{Cu})$, oxides $(\mathrm{Al} 2 \mathrm{O} 3, \mathrm{CuO}$, $\mathrm{TiO} 2$, and $\mathrm{SiO} 2)$, carbides $(\mathrm{SiC})$, nitrides (AlN, $\mathrm{SiN}$ ), and nonmetal (graphite, carbon nanotubes) and the base fluid is usually a conductive fluid, such as water or ethylene glycol. Other base fluids are toluene, oil, other lubricants, biofluids, and polymer solution. Nanoparticles are present up to $5 \%$ volume fraction in nanofluids. The conventional heat transfer fluids are poor conductors of heat. Nanofluids make an edge over them because they have high heat transfer capability. Since these heating/cooling fluids play a vital role in the development of energy efficient heat transfer equipment for energy supply, to raise the thermal conductivity of these fluids, nanosized conducting metal particles are added to them. Most of engineering problems, especially some heat transfer equations are nonlinear, therefore some of them are solved using numerical solution and some are solved using the different analytic method, such as perturbation method (PM),
Therefore, their proper understanding is a must to use them efficiently in modern industry. Applications of nanofluids include microelectronics, fuel cells, and pharmaceutical processes.

Choi and Eastman [1] were the first to propose the term nanofluid that represents the fluid in which nanoscale particles are suspended in the base fluid with low thermal conductivity such as water, ethylene glycol, and oil. In recent years, many researchers have studied and reported nanofluid technology experimentally or numerically in the presence of heat transfer.

Heat transfer of a nanofluid flow which is squeezed between parallel plates was investigated analytically using Homotopy perturbation method (HPM) by Sheikholeslami and Ganji [2]. They reported that Nusselt number has direct relationship with nanoparticle volume fraction, the squeeze number and Eckert number when two plates are separated but it has reverse relationship with the squeeze number when two plates are squeezed.

homotopy perturbation method (HPM), variational iteration method (VIM). Therefore, many different methods have recently introduced some ways to eliminate the small parameter. One of the semiexact methods which does not need small 
parameters is the HPM. The homotopy perturbation method proposed and improved by He [3]. The method yields a very rapid convergence of the solution series in the most cases. The HPM proved its capability to solve a large class of nonlinear problems efficiently, accurately and easily with approximations convergency very rapidly to solution. Usually, few iterations lead to high accuracy solution. This method is employed for many researches in engineering sciences.

HPM was used for solving meany problems such as : nonlinear MHD Jeffery Hamel problem by Moghimi et al. [4]. Mustafa et al. [5] analyzed the effect of squeezing nanofluid flow between parallel plates. Comprehensive account of the use of the HPM successfully to solve problems in fluid mechanics, please see $[6,7]$.

The main objective of this present study is to investigate nanofluid flow and heat transfer between two parallel flat plates without presence of magnetic field. In this study, we have applied Runge-Kutta- Fehlberg fourth-fifth-order method with shooting technique (RKF45) and homotopy perturbation method (HPM), to find the solution of nonlinear differential equations. The effects of governing parameters such as squeeze number, and nanoparticle volume fraction on velocity, and temperature, aswell as on skin-friction coefficient, Nusselt are investigated.

\section{Mathematical Formulation}

We consider an unsteady two-dimensional flow to observe heat andmass transfer of a squeezing nanofluid in the middle of two parallel plates extended infinitely and implanted in a system occupied with nanofluid (water as a base fluid) and (copper as a nanoparticle). The thermophysical properties of the nanofluids are given in Table 1. The distance between two plates is $y=l \sqrt{1-\alpha t}=h(t)$ where $l$ is the initial position

(at time $t=0$ ).

Flow is incompressible with no chemical reaction in system. The graphical model support to the present study has been given in Figure 1.

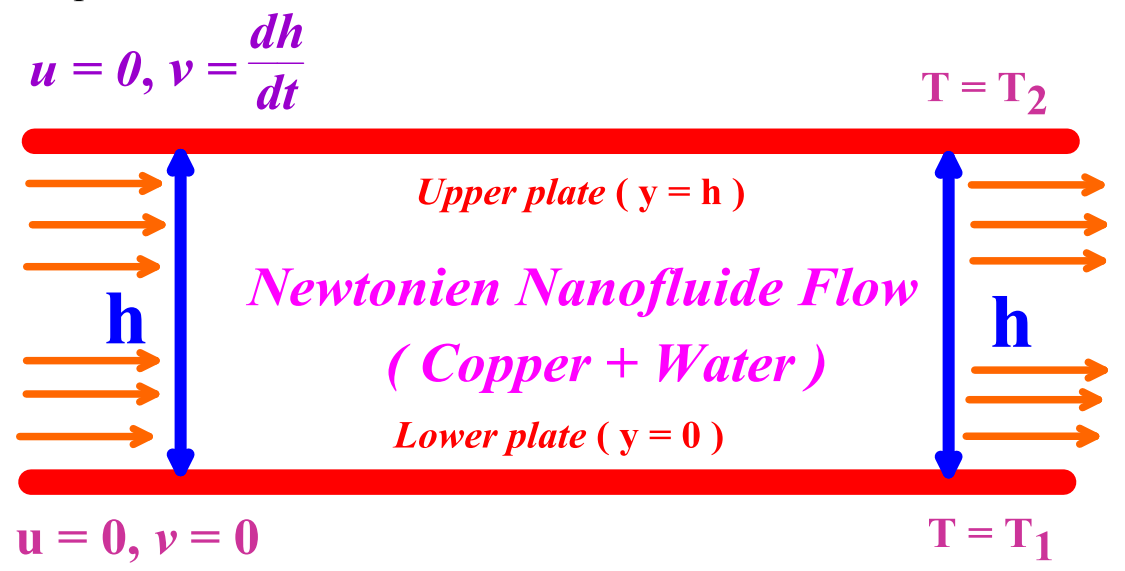

Figure 1 : Geometry of present work.

Table 1 : Thermophysical properties of pure water and nanoparticles.

\begin{tabular}{|c|c|c|c|c|}
\hline & $\rho\left(\mathrm{Kg} / \mathrm{m}^{3}\right)$ & $\mathrm{Cp}(\mathrm{J} / \mathrm{Kg})$ & $\mathrm{K}(\mathrm{W} / \mathrm{mK})$ & $\sigma\left(\mathrm{S} \mathrm{m}^{-1}\right)$ \\
\hline Copper $(\mathrm{Cu})$ & 8933 & 385 & 401 & $5.96 * 10^{7}$ \\
Pure water $\left(\mathrm{H}_{2} \mathrm{O}\right)$ & 997.1 & 4179 & 0.613 & 0.05 \\
\hline
\end{tabular}


The governing equations representing flow are as follows :

$$
\begin{gathered}
\frac{\partial u}{\partial x}+\frac{\partial v}{\partial y}=0 \\
\rho_{n f}\left(\frac{\partial u}{\partial t}+u \frac{\partial u}{\partial x}+v \frac{\partial u}{\partial y}\right) \\
=-\frac{\partial P}{\partial x}+\mu_{n f}\left(\frac{\partial^{2} u}{\partial x^{2}}+\frac{\partial^{2} u}{\partial y^{2}}\right) \\
\rho_{n f}\left(\frac{\partial v}{\partial t}+u \frac{\partial v}{\partial x}+v \frac{\partial v}{\partial y}\right) \\
=-\frac{\partial P}{\partial y}+\mu_{n f}\left(\frac{\partial^{2} v}{\partial x^{2}}+\frac{\partial^{2} v}{\partial y^{2}}\right) \\
\frac{\partial T}{\partial t}+u \frac{\partial T}{\partial x}+v \frac{k_{n f}}{\partial y}\left(\frac{\partial^{2} T}{\partial x^{2}}+\frac{\partial^{2} T}{\partial y^{2}}\right) \\
u \frac{\partial \square}{\partial x}+v \frac{\partial \square}{\partial y}=\frac{\mu_{n f}}{\rho_{n f}}\left(\frac{\partial^{2} \square}{\partial x^{2}}+\frac{\partial^{2} \square}{\partial y^{2}}\right)
\end{gathered}
$$

equation (5) is called the vorticity equation is obtained by doing : $\frac{\partial(3)}{\partial x}-\frac{\partial(2)}{\partial y}$ with $\square=\frac{\partial v}{\partial x}-$ $\frac{\partial u}{\partial y}$

where $u$ and $v$ are the velocity components in the $\mathrm{x}$ and $\mathrm{y}$ directions, respectively, $\rho_{n f}$ is the effective density of the nanofluid, $\mu_{n f}$ is the effective dynamic viscosity of the nanofluid, $\left(\rho c_{p}\right)_{n f}$ is the heat capacity of the nanofluid and $k_{n f}$ is the thermal conductivity of the nanofluid, are given as :

$$
\begin{gathered}
\rho_{n f}=(1-\phi) \rho_{f}+\phi \rho_{p} \\
(\rho C p)_{n f}=(1-\phi)(\rho C p)_{f}+\phi(\rho C p)_{p} \\
f^{\prime \prime \prime \prime}-S \frac{A_{1}}{A_{2}}\left(3 f^{\prime \prime}+\eta f^{\prime \prime \prime}+f^{\prime} f^{\prime \prime}-f f^{\prime \prime \prime}\right)=0 \\
\mu_{n f}=\frac{\mu_{f}}{(1-\phi)^{2.5}}
\end{gathered}
$$

$$
K_{n f}=\frac{K_{s}+2 K_{f}-2 \phi\left(K_{f}-K_{s}\right)}{K_{s}+2 K_{f}+2 \phi\left(K_{f}-K_{s}\right)} K_{f}
$$

The associated boundary conditions for the time dependent squeezing flow are as follows :

$$
\begin{gathered}
u=0, v=0, T=T_{1} \quad \text { en } \quad y=0 \\
u=0, v=\frac{d h}{d t}, T=T_{2} \quad \text { en } \quad y=h(t)
\end{gathered}
$$

In the Eq. (11), $v=\frac{d h}{d t}$ indicate the velocity with which upper plate is moving towards or away from the lower plate which is kept at a distance $y=0$ from the upper plate at $y=h(t)$. Further, the value of the $v$ is exam as $\left[\frac{-\alpha l}{2(1-\alpha t)^{\frac{1}{2}}}\right]$.

However, Eqs. (4)-(5) along with conditions (10) and (11) governing the nanofluid squeezing flow are coupled highly nonlinear in nature and are not amenable to any analytical methods. Hence, in the system of partial differential equations, Eqs. (4)(5) are reduced to ordinary differential equations by using the following similarity transf ormations :

$$
\begin{gathered}
\eta=\frac{y}{l(1-\alpha t)^{\frac{1}{2}}}=\frac{y}{h(t)} \\
u=\frac{\alpha x}{2(1-\alpha t)} f^{\prime}(\eta) \\
v=\frac{-\alpha l}{2(1-\alpha t)^{\frac{1}{2}}} f(\eta) \\
\Theta=\frac{T-T_{2}}{T_{1}-T_{2}}
\end{gathered}
$$

and substituting Eq. (12) in Eqs. (5) and (6), we obtain the reduced governing equations :

- The nonlinear fourth-order ordinary differential equation for the momentum equation : 
- The second order differential equation for the energy equation :

where $A_{1}, A_{2}$ and $A_{3}$ are dimensionless constants defined as follows :

$$
\begin{gathered}
A_{1}=\frac{\rho_{n f}}{\rho_{f}} \\
A_{2}=\frac{\mu_{n f}}{\mu_{f}} \\
A_{3}=\frac{K_{n f}}{K_{f}} \\
A_{4}=\frac{\left(\rho c_{p}\right)_{n f}}{\left(\rho c_{p}\right)_{f}}
\end{gathered}
$$

The boundary conditions (10) in the terms of similarity variables (11) become :

$f^{\prime}(0)=0, f(0)=0, \Theta=1$ at $\eta=0$

$f^{\prime}(1)=0, f(1)=1, \Theta=0 \quad$ at $\quad \eta=1$

where $S=\frac{\alpha l^{2}}{2 \mathrm{v}_{f}} \quad$ is the squeeze number, $\operatorname{Pr}=\frac{(\rho C p)_{f}}{K_{f}} \mathrm{v}_{f}$ is the Prandtl number.

The physical quantities of interest are the skinfriction coefficient $C_{f}$, the Nusselt number $N_{u}$, defined as :

$$
\begin{gathered}
c_{f}=\frac{\tau_{w}}{\rho_{n f} \mathrm{v}_{w}} \\
N_{u}=\frac{l q_{w}}{k_{f}\left(T_{1}-T_{2}\right)}
\end{gathered}
$$

Where :

$$
\begin{gathered}
\tau_{w}=\mu_{n f}\left(\frac{\partial u}{\partial y}\right)_{y=0} \\
\left.q_{w}=-k_{n f} \frac{\partial T}{\partial y}\right)_{y=0}
\end{gathered}
$$

Using (7) and (11) in (10), we get :

$$
C_{f}^{*}=\frac{x^{2}}{l^{2}}(1-\alpha t) R_{e_{x}} C_{f}=\frac{f^{\prime \prime}(0)}{(1-\phi)^{2.5} A_{1}}
$$

$$
\Theta^{\prime \prime}+\operatorname{SPr} \frac{A_{4}}{A_{3}}\left(f \Theta^{\prime}-\mathrm{\eta} \Theta^{\prime}\right)=0
$$

$$
N_{u_{x}}^{*}=\sqrt{1-\alpha t} N_{u_{x}}=-A_{3} \Theta^{\prime}(0)
$$

Where $R_{e_{x}}=\frac{\alpha l^{5}}{2 x^{3}(1-\alpha t)^{\frac{1}{2}} \mathrm{v}_{f}}$ the local Reynolds number.

\section{Basic Idea of the Homotopy Perturbation Method}

To illustrate the basic ideas of this method, we consider the following equation :

$$
A(u)-f(r)=0, \quad r \in \Omega
$$

with the boundary condition of :

$$
B\left(u, \frac{\partial U}{\partial n}\right)=0, \quad r \in \Gamma
$$

where $A$ is a general differential operator, $B$ a boundary operator, $f(r)$ a known analytical function and $\Gamma$ is the boundary of the domain $\Omega$. $A$ can be divided into two parts which are $L$ and $N$, where $L$ is linear and $N$ is nonlinear. Eq. (16) can therefore be rewritten as follows :

$$
L(u)+N(u)-f(r)=0
$$

Homotopy perturbation structure is shown as follows :

$$
\begin{gathered}
H(v, p)=(1-p)\left[L(v)-L\left(u_{0}\right)\right]+p[L(v) \\
+N(v)-f(r)]=0
\end{gathered}
$$

Where :

$$
v(r, p): \Omega \times[0,1] \rightarrow R
$$

In Eq. (22), $p \in[0,1]$ is an embedding parameter and $0 \mathrm{u}$ is the first approximation that satisfies the boundary condition. We can assume that the solution of Eq. (21) can be written as a power series in $\mathrm{p}$, as following : 
$v=v_{0}+p v_{1}+p^{2} v_{2}+p^{3} v_{3}+\cdots$

and the best approximation for solution is :

$$
u=\lim _{p \rightarrow 1} v=v 0+v 1+v 2+\cdots
$$

\section{Implementation of the method}

According to the so-called homotopy-perturbation method (HPM), we construct a homotopy suppose the solution of Eq. (17) has the form :

$$
\begin{aligned}
& (1-p)\left[f^{\prime \prime \prime \prime}-f_{0}{ }^{\prime \prime \prime}(0)\right] \\
& +p\left[f^{\prime \prime \prime \prime}+S A_{1}(1\right. \\
& -\phi)^{2.5}\left[3 f^{\prime \prime}+\eta f^{\prime \prime \prime}+f^{\prime} f^{\prime \prime}\right. \\
& \left.\left.-f f^{\prime \prime \prime}\right]\right]=0 \\
& (1-p)\left[\Theta^{\prime \prime}-\Theta_{0}{ }^{\prime \prime}(0)\right] \\
& +p\left\{\Theta^{\prime \prime}\right. \\
& \left.+S \operatorname{Pr} \frac{A_{2}}{A_{3}}\left[f \Theta^{\prime}-\eta \Theta^{\prime}\right]\right\}=0
\end{aligned}
$$

We consider $f$ and $\theta$ as follows :

$$
\begin{aligned}
& f(\eta)=f_{0}(\eta)+f_{1}(\eta)+f_{2}(\eta)+f_{3}(\eta)+\cdots \\
& =\sum_{i=0}^{N} f_{i}(\eta) \\
& \theta(\eta)=\theta_{0}(\eta)+\theta_{1}(\eta)+\theta_{2}(\eta)+\theta_{3}(\eta)+\cdots \\
& =\sum_{i=0}^{N} \theta_{i}(\eta)
\end{aligned}
$$

with substituting $f, \theta$ from equations (20 - 21) into equations (18-19) and some simplification and rearranging based on powers of $p$ - terms, we have :

$$
\begin{gathered}
p^{0}: \\
f^{i v}=0, \\
\theta_{0}^{\prime \prime}=0,
\end{gathered}
$$

And boundary conditions are :

$$
\begin{gathered}
f(0)=0, f^{\prime}(0)=0, f(1)=1, f^{\prime}(1)=0, \\
\theta(0)=1 \text { et } \theta(1)=0
\end{gathered}
$$

$$
P^{1}:
$$

$$
\begin{gathered}
f_{1}^{i v}-1.102087119 S\left(\mathrm{\eta} f_{0}^{\prime \prime \prime}+3 f_{0}^{\prime \prime}+f_{0}^{\prime} f_{0}^{\prime \prime}\right. \\
\left.-f_{0} f_{0}^{\prime \prime \prime}\right)=0
\end{gathered}
$$

$$
\Theta_{1}^{\prime \prime}+0.9201899729 \operatorname{Pr} S\left(\Theta_{0}^{\prime} f_{0}-\eta \Theta_{0}^{\prime}\right)=0
$$

And boundary conditions are :

$$
\begin{aligned}
& f(0)=0, f^{\prime}(0)=0, f(1)=0, f^{\prime}(1)=0, \\
& \theta(0)=0 \text { et } \theta(1)=0
\end{aligned}
$$

Solving equations (22) and (24) with boundary conditions, we have :

$$
\begin{gathered}
f_{0}=-2 \eta^{3}+3 \eta^{2} \Theta_{0}=-\eta+1 \\
f_{1}=\frac{254789641}{45623178} S\left(\frac{1}{875} \eta^{7}-\frac{1}{68} \eta^{6}\right. \\
\left.-\frac{1}{97} \eta^{5}+\frac{1}{74} \eta^{4}\right) \\
-\frac{45879621}{5879641} \eta^{3} S \\
+\frac{57896412}{25896413} \eta^{2} S \\
\theta_{1}=-\frac{8569742301}{15419872} S P r\left(\frac{1}{45} \eta^{5}-\frac{1}{82} \eta^{4}\right. \\
\left.+\frac{1}{78} \eta^{3}\right) \\
+\frac{254789620}{15478963} S P r \eta
\end{gathered}
$$


The terms $f_{i}(\eta), \Theta_{i}(\eta)$ when $i>2$ are too large that is mentioned graphically. The solution of this equation, when $p \rightarrow 1$, will be as follows:

$$
\begin{aligned}
f(\mathrm{\eta})=f_{0}(\mathrm{\eta}) & +f_{1}(\mathrm{\eta})+f_{2}(\mathrm{\eta})+f_{3}(\mathrm{\eta})+\cdots \\
& =\sum_{i=0}^{N} f_{i}(\mathrm{\eta}) \\
\theta(\mathrm{\eta})=\theta_{0}(\mathrm{\eta}) & +\theta_{1}(\mathrm{\eta})+\theta_{2}(\mathrm{\eta})+\theta_{3}(\mathrm{\eta})+\cdots \\
& =\sum_{i=0}^{N} \theta_{i}(\mathrm{\eta})
\end{aligned}
$$

\section{Conclusions}

In this paper, Runge-Kutta and HPM Method is applied to solve the problem of the magnetohydrodynamic squeezing flow of nanofluid between parallel plate. The effects of active parameters such as squeeze number, nanoparticle volume fraction are investigated.

To verify the present analytical solution, we compared our results with results given by using Runge-Kutta. They are in an excellent agreement as they have been demonstrated in Table 2 and.

\section{Table 3.}

Figure 2 shows the effect of increasing the squeeze number $S$ on the profiles of the velocity the latter increasing to the movable plate is in the middle of the initial distance between the plates, then it decreases slightly and tends towards zero when the two plates are glued together, and we also observe that the speed decreases with increasing values of $S$.

It can easily be seen in Figure 3 that the value of the temperature near the bottom surface of the plate decreases steadily with the increase of the value of $S$, and that as we move away from the lower surface of the plate, this value increases.

Figure 5 shows the effect of increasing the volume fraction on the velocity profile which increases to the moving plate in the middle of the initial distance between the plates, then decreases slightly and tends to zero when the two plates are glued together, and we also observe that the speed decreases with increasing values of $\varphi$.

Table 2 : Comparison between (HMP) and Runge - Kutta for $f^{\prime}(\eta)$.
Figure 4 shows the effect of increasing the volume fraction on the temperature profile Given the previous temperature curve, there is practically no change in the temperature profile when $\varphi$ varies, but there is an effect well shown in the Table 4, initially present an increase in the values of the volume fraction of the nanoparticles $\varphi$, the temperature decreases, and after a fixed distance from the lower surface of the plate, it increases slightly.

From Table 5 it is evident that the coefficient of friction and the Nusselt number are proportional to $S$, and it is observed that the parameters $C_{f}^{*}$ increase with increasing values of $S$ and $N_{u}^{*}$ decrease as $S$ increases.

In Table 6 it is evident that the coefficient of friction and the Nusselt number are inversely proportional to $\varphi, C_{f}^{*}$ and $N_{u}^{*}$ decreases as $\varphi$ increases.

\begin{tabular}{|c|c|c|}
\hline \multirow{2}{*}{$\eta$} & \multicolumn{2}{|c|}{$f^{\prime}(\eta)$} \\
\cline { 2 - 3 } & \multicolumn{2}{|c|}{$\varphi=0.02, \quad S=1, N=4$} \\
\cline { 2 - 3 } & HPM & Runge-Kutta \\
\hline
\end{tabular}




\begin{tabular}{|c|c|c|}
\hline 0.0 & 0.0 & 0.0 \\
\hline 0.2 & 0.9724211464 & 0.9724213434 \\
\hline 0.4 & 1.421472921 & 1.4214727055 \\
\hline 0.6 & 1.42147292 & 1.4214727055 \\
\hline 0.8 & 0.972421143 & 0.9724213434 \\
\hline 1.0 & 0.0 & 0.0 \\
\hline
\end{tabular}

Table 3 : Comparison between (HMP) and Runge - Kutta for $\theta^{\prime}(\eta)$.

\begin{tabular}{|c|c|c|}
\hline \multirow{2}{*}{$\eta$} & \multicolumn{2}{|c|}{$\theta^{\prime}(\eta)$} \\
\cline { 2 - 3 } & HPM $\varphi=0.02, \quad S=1, N=4$ \\
\cline { 2 - 3 } & 1.0 & Runge - Kutta \\
\hline 0.0 & 0.8129480713 & 1.0 \\
\hline 0.2 & 0.6076612514 & 0.8129480503 \\
\hline 0.4 & 0.3923387482 & 0.6076612254 \\
\hline 0.6 & 0.1870519301 & 0.3923387745 \\
\hline 0.8 & 0.0 & 0.1870519496 \\
\hline 1.0 & & 0.0 \\
\hline
\end{tabular}

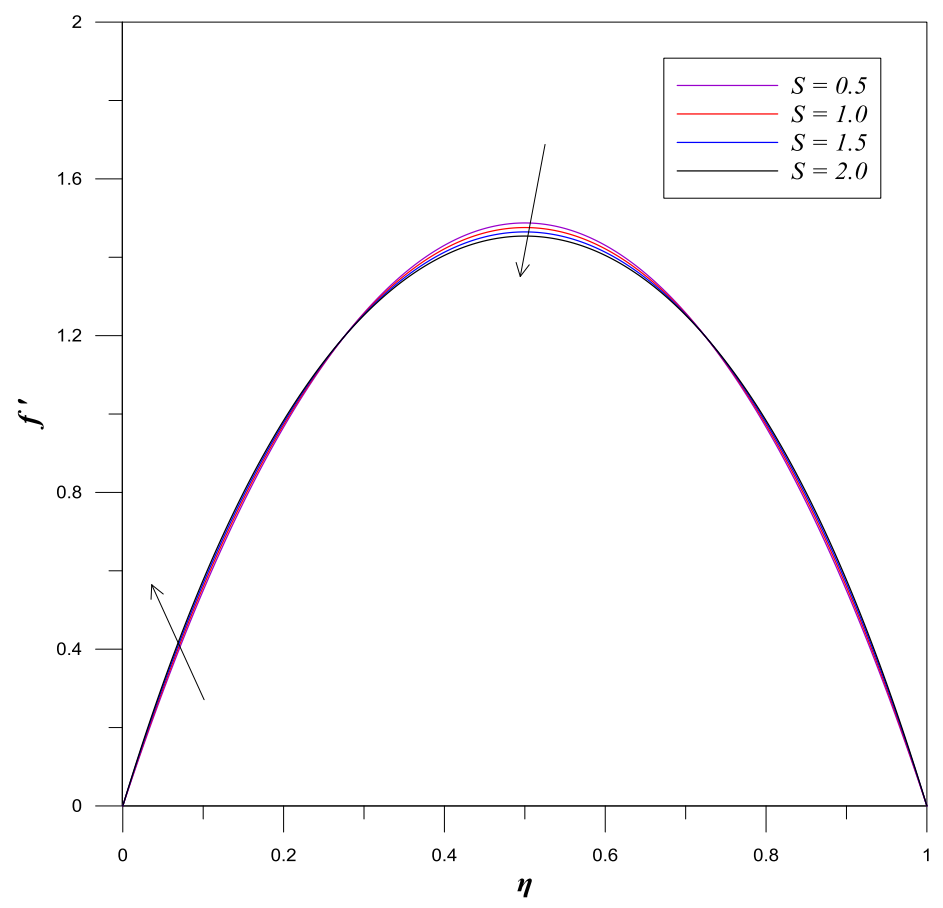

Figure 2 : velocity profile for different squeeze number values $(S)$. 


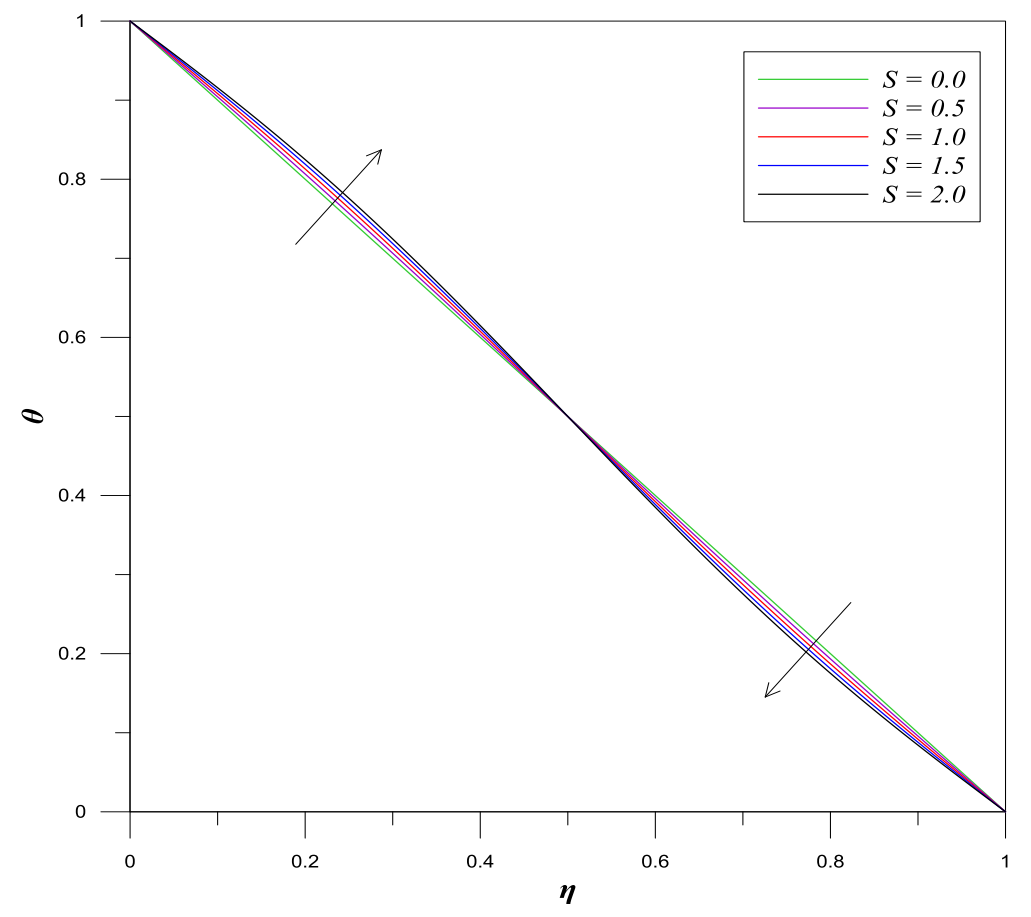

Figure 3 : temperature profile for different squeeze number values $(S)$.

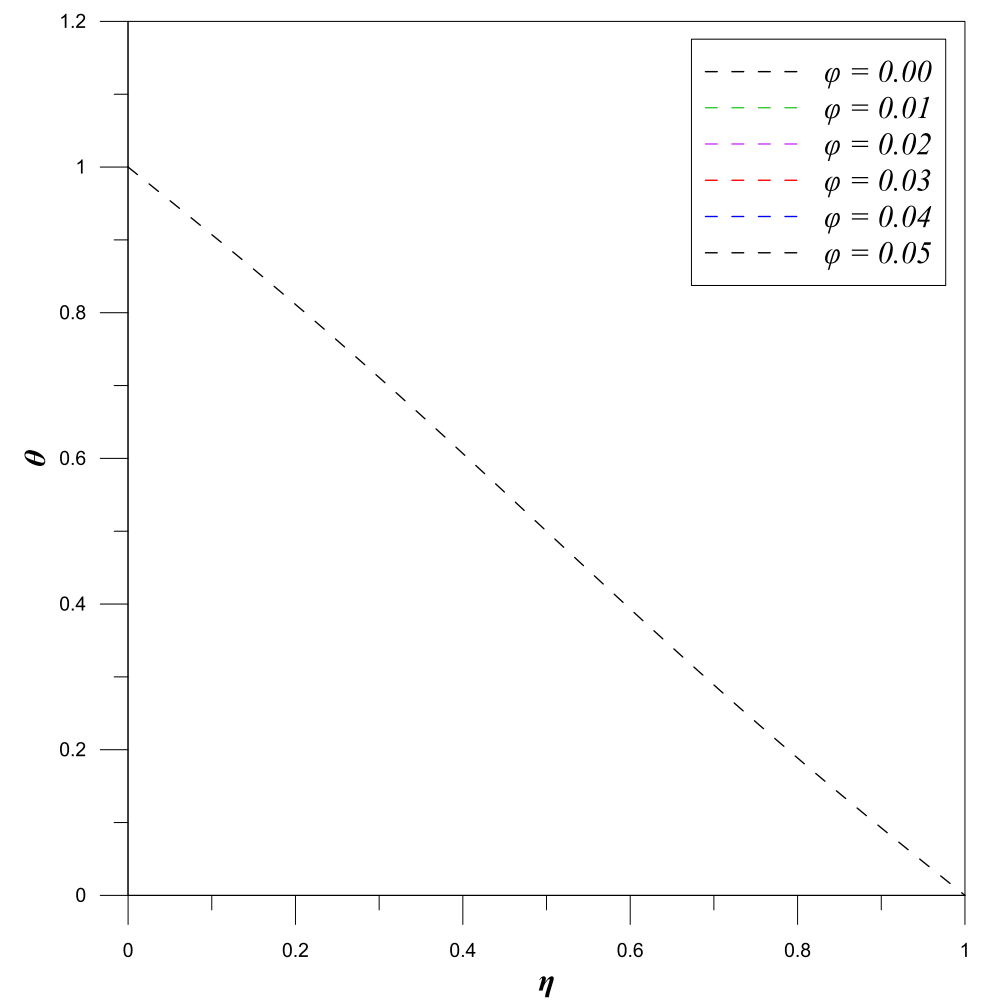

Figure 4 : temperature profile for different volume fraction values $(\varphi)$. 


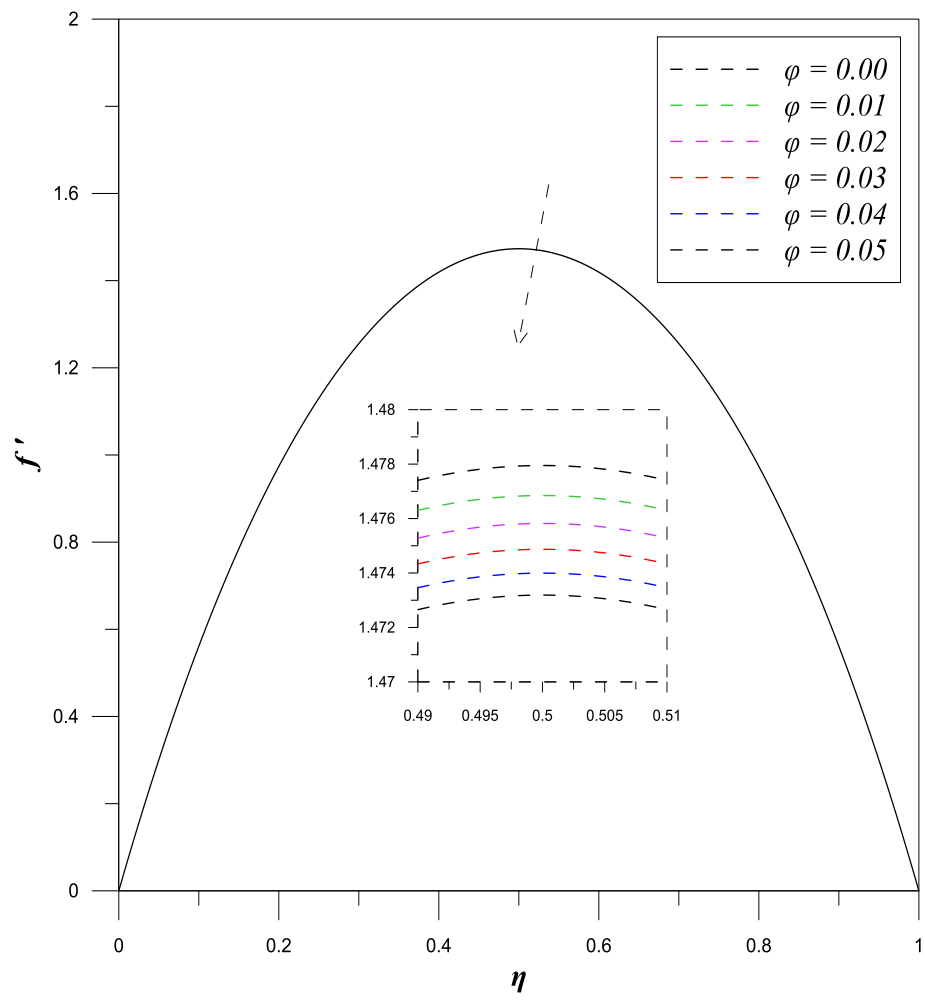

Figure 5 : velocity profile for different volume fraction values $(\varphi)$.

Table 4 : Temperature value for different values of $\varphi$.

\begin{tabular}{|c|c|c|c|c|c|c|}
\hline \multirow{3}{*}{$\eta$} & \multicolumn{9}{|c|}{$N=3=1$} & \multicolumn{2}{c|}{} \\
\cline { 2 - 7 } & $\varphi=0.00$ & $\varphi=0.01$ & $\varphi=0.02$ & $\varphi=0.03$ & $\varphi=0.04$ & $\varphi=0.05$ \\
\cline { 2 - 8 } & $\Theta$ & $\Theta$ & $\Theta$ & $\Theta$ & $\Theta$ & \multirow{2}{*}{$\Theta$} \\
\hline 0 & 1 & 1 & 1 & 1 & 1 & 1 \\
\hline 0.1 & 0.9089796135 & 0.9086112279 & 0.9079192365 & 0.9082580644 & 0.9075939228 & 0.9072813605 \\
\hline 0.2 & 0.815073728 & 0.8135108681 & 0.8124090419 & 0.8129481537 & 0.8118921319 & 0.8113961267 \\
\hline 0.3 & 0.7137466214 & 0.7131637223 & 0.7120736244 & 0.7126065942 & 0.7115633264 & 0.7110743294 \\
\hline 0.4 & 0.6083624809 & 0.6080035292 & 0.6073333522 & 0.6076608342 & 0.6070201245 & 0.6067202682 \\
\hline 0.5 & 0.5000000001 & 0.5000000001 & 0.4999999997 & 0.5000000004 & 0.4999999996 & 0.4999999996 \\
\hline 0.6 & 0.3916375189 & 0.391996471 & 0.3926666473 & 0.3923391662 & 0.3929798746 & 0.3932797318 \\
\hline 0.7 & 0.2862533788 & 0.2868362781 & 0.2879263752 & 0.2873934062 & 0.2884366725 & 0.2889256704 \\
\hline 0.8 & 0.1859012994 & 0.1864891321 & 0.187590958 & 0.1870518467 & 0.1881078676 & 0.1886038731 \\
\hline 0.9 & 0.0910203874 & 0.0913887706 & 0.187590958 & 0.0917419333 & 0.0924060768 & 0.09271864 \\
\hline 1 & 0 & 0 & 0 & 0 & 0 & 0 \\
\hline
\end{tabular}


Table 5 : Variation of $C_{f}^{*}$ and $N_{u}^{*}$ for different values of S.

\begin{tabular}{|c|c|c|}
\hline \multirow{2}{*}{$S$} & \multicolumn{2}{|c|}{$N=4, \varphi=0.02$} \\
\cline { 2 - 3 } & $C_{f}^{*}$ & $N_{u}^{*}$ \\
\hline 0.5 & 5.61973 & 0.88071 \\
\hline 1 & 5.78950 & 0.84032 \\
\hline 1.5 & 5.95406 & 0.80209 \\
\hline 2 & 6.11394 & 0.76587 \\
\hline
\end{tabular}

Table 6: Variation of $C_{f}^{*}$ and $N_{u}^{*}$ for different values of $\varphi$.

\begin{tabular}{|c|c|c|}
\hline \multirow{2}{*}{$\varphi$} & \multicolumn{2}{|c|}{$N=3, S=1$} \\
\cline { 2 - 3 } & $C_{f}^{*}$ & $N_{u}^{*}$ \\
\hline 0 & 6.34632 & 0.90222 \\
\hline 0.01 & 6.04486 & 0.87082 \\
\hline 0.02 & 5.78950 & 0.84032 \\
\hline 0.03 & 5.57157 & 0.81069 \\
\hline 0.04 & 5.38450 & 0.78190 \\
\hline 0.05 & 5.22323 & 0.75391 \\
\hline
\end{tabular}




\section{Nomenclature}

$\begin{array}{cl}S & \text { Squeeze number } \\ f^{\prime}, f & \text { Dimensionless velocity } \\ \theta & \text { Dimensionless temperature } \\ P & \text { Pressure term } \\ P r & \text { Prandtl number } \\ T & \text { Temperature }(\mathrm{K}) \\ x, y & \text { Cartesian coordinates } \\ u & \text { Velocity component in } \mathrm{x} \\ & \text { direction }(\mathrm{m} / \mathrm{s}) \\ v & \text { Velocity component in } \mathrm{y} \\ & \text { direction (m/s) } \\ N u & \text { Nusselt number } \\ C f & \text { Skin fraction coefficient } \\ k & \text { Thermal conductivity } \\ A 3, A 4, A 5 & \text { Dimensionless constants }\end{array}$

\section{Greek symbols}

$\begin{array}{ll}\alpha & \text { Rate of squeezing } \\ \eta & \text { Dimensionless variable } \\ \varphi & \text { Solid volume fraction } \\ \rho & \text { Density } \\ \mu & \text { Dynamic viscosity } \\ \mathrm{v} & \text { Kinematic viscosity }\end{array}$

\section{Subscripts}

$\begin{aligned} n f & \text { Nanofluid } \\ s & \text { Nano-solid-particles } \\ f & \text { Base fluid }\end{aligned}$

\section{References}

[1] S. U. S. Choi and J. A. Eastman, "Enhancing thermal conductivity of fluids with nanoparticle," in Proceedings of the International Mechanical Engineering Congress and Exhibition, vol. 231, pp. 99-105, San Francisco, Calif, USA, November 1995.

[2] M. Sheikholeslami, D.D. Ganji, Heat transfer of $\mathrm{Cu}$-water nanofluid flow between parallel plates,

Powder Technology 235 (2013) 873-879.

[3] J.H. He, Comparison of homotopy perturbation method and homotopy analysis method, Appl. Math. Comput., 156, (2004), 527-539.

[4] S.M. Moghimi, D.D. Ganji, H. Bararnia ,M. Hosseini, M. Jalaal, Homotopy perturbation method for nonlinear MHD JefferyHamel problem. Computers and Mathematics with Applications, 61, (2011)2213-2216.

[5] M. Mustafa, T. Hayat, S. Obaidat, On heat and mass transfer in the unsteady squeezing flow between parallel plates, Meccanica, DOI 10.1007/s11012-012-9536-3.

[6] A. El Harfouf, A. Wakif, S.Hayani Mounir, Sensor Letters, 17(2019)1-9, doi :

10.1166/sl.2020.4169.

[7] EL HARFOUF, AMINE, WAKIF, A., et MOUNIR, S. Hayani. Analytical resolution on Unsteady nanofluid flow problem between two parallel plates a solution by using a Semianalytical Method. Applied Journal of Environmental Engineering Science, 2020, vol. 6, no 3, p. 6-3 (2020) 325-337.

\section{Creative Commons Attribution License 4.0 (Attribution 4.0 International, CC BY 4.0)}

This article is published under the terms of the Creative Commons Attribution License 4.0

https://creativecommons.org/licenses/by/4.0/deed.en_US 